PROCEEDINGS OF THE

AMERICAN MATHEMATICAL SOCIETY

Volume 131, Number 1, Pages 285-290

S 0002-9939(02)06523-1

Article electronically published on May 22, 2002

\title{
MARKET COMPLETENESS: A RETURN TO ORDER
}

\author{
GEORGE STOICA
}

(Communicated by Claudia M. Neuhauser)

\begin{abstract}
We investigate market completeness with respect to the topology generated by the order relation on a linear lattice of functions describing, in a two-times economy, the associated cash flow space. The study is leading us towards alternative definitions for market completeness, in terms of trading strategy operators and approximate convergent martingales spanning on such linear lattices.
\end{abstract}

\section{INTRODUCTION}

In solving the Artzner and Heath paradox (an economy with complete markets and a multiplicity of martingale measures), Jarrow, Jin and Madan presented in [3] a resolution that lies in noting that market completeness is with respect to a topology on the space of cash flows $L^{\infty}$ based on a probability field $(\Omega, \mathcal{K}, P)$; by their version of the second fundamental theorem of asset pricing, the latter is always equivalent to uniqueness of the signed state price density in the dual topology. Moreover (see [1), existence of a state price density is equivalent to "no weak arbitrage opportunities", and existence of a positive state price density is equivalent to "no weak arbitrages with free disposal".

As remarked in [2], [5] and the references therein, the space of cash flows need not be (the entire) $L^{\infty}(\Omega, \mathcal{K}, P)$ but, essentially, a subspace of functions for which the vectorial and latticial operations are compatible, that is, a linear lattice. The aim of this paper is to investigate market completeness on fairly general linear lattices, in the topology generated by the order relation. We present two possible definitions for completeness; the main tools employed are: an appropriate definition of the trading strategy operator, and an order convergent martingales spanning argument.

\section{ORDER COMPLETE MARKETS}

In a two-times economy, let us consider a linear lattice of functions $\mathbf{C}=(\mathbf{C}, \vee, \wedge)$ to be the space of cash flows, and let us denote the space of admissible trading strategies by $\mathbf{Y}$, where $y=\left(y_{\alpha}\right)_{\alpha \in \mathbb{R}} \in \mathbf{Y}$, with $y_{\alpha} \in \mathbf{C}$ for all $\alpha \in \mathbb{R}$, defines the total position in all assets with index position less than or equal to $\alpha$. For each $\alpha$, ownership of the asset gives the holder the claim to a cash flow $c_{\alpha} \in \mathbf{C}$.

Received by the editors February 19, 2001 and, in revised form, August 18, 2001

2000 Mathematics Subject Classification. Primary 91B24, 91B70; Secondary 60G42, 46A40.

The author is indebted to the Probability Editor, whose remarks improved the present version of the note. 
For each $y \in \mathbf{Y}$, we define the cash flow obtained by the admissible trading strategy as $\Phi(y)$, where the trading strategy (linear) operator $\Phi: \mathbf{Y} \rightarrow \mathbf{C}$ is defined, following a Freudenthal-type spectral representation in linear lattices (see [4], Chapter 6), by

$$
\Phi(y)=(0)-\lim _{\left\|\Delta_{n}\right\| \rightarrow 0} \sum_{i \in \mathbb{Z}} c_{r_{i}^{n}}\left(y_{u_{i+1}^{n}}-y_{u_{i}^{n}}\right),
$$

if the above (o)-limit, taken in the order topology on $\mathbf{C}$, exists. The above Stieltjestype sums are computed with respect to a sequence of partitions $\Delta_{n}=\left(u_{i}^{n}\right)_{i \in \mathbb{Z}}$ of $\mathbb{R}$, where $u_{i}^{n} \leq r_{i}^{n}<u_{i+1}^{n}$ for $i \in \mathbb{Z}$ are sample points, and $\left\|\Delta_{n}\right\|$ is the norm of the partition $\Delta_{n}$. Recall that $x=(\mathrm{o})-\lim _{n \rightarrow+\infty} x_{n}$ whenever there is a sequence $\left\{v_{n}\right\}$ in $\mathbf{C}$ such that $\left|x_{n}-x\right| \leq v_{n}$ for any $n$, and $v_{n} \searrow 0$ as $n \rightarrow+\infty$; here, $\searrow$ means decreasing towards, $|a|=a_{+}+a_{-}$, and $a_{+}, a_{-}$are the positive and negative part of $a$, respectively.

Remark 1. The particular definition given in [3] for the trading operator $\Phi$, that is, as an abstract integral with respect to a positive measure, cannot be used in the above general context: our $\Phi$, being a Stieltjes-type integral operator, need not be (o)-sequentially continuous. Hence, the special locally convex topology on $\mathbf{C}$ from [3], generated by their continuous linear operator $\Phi$, will be replaced in our setting by the order topology and, as such, we are lead towards the following.

Definition 1. The market is called complete with respect to the order topology, in short (o)-complete, if for all $c \in \mathbf{C}$, there is a $y \in \mathbf{Y}$ such that $\Phi(y)=c$.

To state the results of our paper, we shall need the following facts about lattices. The subset $\mathbf{M}$ is called a projection band in $\mathbf{C}$ (see [4]) if $\mathbf{C}$ is the direct sum of $\mathbf{M}$ and $\mathbf{M}^{\perp}$, where $\mathbf{M}^{\perp}:=\{y \in \mathbf{C}$ such that $|y| \wedge|x|=0$ for $x \in \mathbf{M}\}$ is the order orthogonal of $\mathbf{M}$. The projection band $\mathbf{M}$ generates a (linear, order bounded) projection operator, denoted by $M$, with the special property that $0 \leq M(x) \leq x$ for $0 \leq x \in \mathbf{C}$. Similarly one defines the operators $M^{\perp}$ and $M^{\perp \perp}$, the latter being generated by the second order orthogonal $\mathbf{M}^{\perp \perp}:=\left(\mathbf{M}^{\perp}\right)^{\perp}$. It is easy to see that $\mathbf{M}^{\perp \perp}$ is always a projection band for any subset $\mathbf{M}$ of $\mathbf{C}$; in particular, we shall denote by $x^{\perp \perp}$ the projection operator generated by the projection band $\{x\}^{\perp \perp}$. A linear lattice is called countably order complete if any countable bounded subset has $\vee$ and $\wedge$; an element is called a weak unit of $\mathbf{C}$ if its order orthogonal is $\{0\}$.

Theorem 1. Suppose $\mathbf{C}$ is a countably order complete linear lattice with weak unit. If $c_{\alpha}$ is a monotone increasing and continuous function such that $\lim _{\alpha \rightarrow \pm \infty} c_{\alpha}= \pm \infty$, then the market is (o)-complete in the sense of Definition 1.

Indeed, we shall prove in the last section that, if we denote by $\mathbf{1}$ the weak unit in $\mathbf{C}$, then $y=\left(y_{\alpha}\right)_{\alpha \in \mathbb{R}} \in \mathbf{Y}$ defined by

$$
y_{\alpha}:=\left(c_{\alpha} \cdot \mathbf{1}-c\right)_{+}^{\perp \perp}(\mathbf{1})
$$

is the right candidate for (o)-completeness in Theorem 1.

Now, let us focus on two important consequences of (o)-completeness. As mentioned in the Introduction, the second fundamental theorem of asset pricing relates market completeness with respect to a topology to uniqueness of (signed) state price densities. In our setting, let us denote by $\pi$ the market price function, that is, $\pi: \mathbb{R} \rightarrow \mathbf{Y}, \Phi \circ \pi=c$. Theorem 1 states the inversibility of the operator $\Phi$, hence 
we may write $\pi_{\alpha}=\Phi^{-1} \circ c_{\alpha}$ and, further, we may identify the state price density by $\Phi^{-1}$. By formula (2) it obviously follows that the components of $y=\left(y_{\alpha}\right)_{\alpha \in \mathbb{R}}$ are all positive and, as $y=\Phi^{-1}(c)$ for any given $c \in \mathbf{C}$, the above facts prove the following.

Corollary 1. Under the hypothesis of Theorem 1, the state price density is unique with respect to the order topology on $\mathbf{C}$ and has all components positive, hence the market has the "no weak arbitrage opportunities" and the "no weak arbitrages with free disposal" properties.

As mentioned in [3], "markets do not have the arbitrage-free property just because they happen to complete". Corollary 1 above says that we are dealing with a "well-posed setup" here, yielding markets that are both complete and arbitrage-free.

On a countably order complete linear lattice $\mathbf{C}$ with weak unit $\mathbf{1}$, it is appropriate to define the payoff of a put option (or of a call option) on $c \in \mathbf{C}$, with exercise price $k \in \mathbb{R}$, by $(k \cdot \mathbf{1}-c)_{+}^{\perp \perp}(\mathbf{1})$ or $(c-k \cdot \mathbf{1})_{+}^{\perp \perp}(\mathbf{1})$, respectively. As such, the $y_{\alpha}$ 's in formula (2) become payoffs of put options, and using Theorem 1 and Definition 1 of (o)-complete markets, we obtain

Corollary 2. Under the hypothesis of Theorem 1, any traded cash flow $c \in \mathbf{C}$ can be written as the (o)-limit of put options (or, considering complementary latticial projection bands, of call options).

The latter extends previous results on Ross-type approximate spanning (see [2], 5]) where, to prove market completeness, the traded cash flows space was supposed to be closed under pointwise convergence of monotone sequences, or the markets were supposed to include all call options on the traded cash flows. Our setting avoids these assumptions and, as such, instead of writing cash flows as almost sure limit of call options, we now use (o)-limits.

Remark 2. Corollaries 1 and 2 are no longer valid in the weak topologies on a linear space $\mathbf{C}$, where additional conditions for market completeness are required (see, for example, Assumptions A and B in [3]).

\section{HOW FAIR ARE ORDER COMPLETE MARKETS?}

As a measure of fairness, it is expected that the notion of complete market related to a given structure (algebraic, topological, ordered, etc.) on the cash flow space be intimately related to martingales; more precisely, the degree of completeness should be reflected in the degree of density of conditional expectation-type martingales within the class of all martingales with respect to the given structure. To describe the extent to which the above fact is true in our latticial setting, we shall use the terminology introduced in our papers [6]-7], where the conditional expectation-type martingales (or the uniformly integrables ones) are the so-called "regular martingales", and are defined as follows.

A filtration on a linear lattice $\mathbf{C}$ is an increasing family $\left(\mathbf{M}_{n}\right)_{n \geq 1}$ of projection bands. A process $\left(x_{n}\right)_{n \geq 1} \subset \mathbf{C}$ is called adapted with respect to $\left(\mathbf{M}_{n}\right)_{n \geq 1}$ iff $x_{n} \in \mathbf{M}_{n}$ for $n \geq 1$. An adapted process $\left(x_{n}\right)_{n \geq 1} \subset \mathbf{C}$ is called (o)-martingale with respect to $\left(\mathbf{M}_{n}\right)_{n \geq 1}$ iff $x_{n}=M_{n}\left(x_{n+1}\right)$ for $n \geq 1$. A regular (o)-martingale is a process of the form $\left(M_{n}(x)\right)_{n \geq 1}$ for fixed $x \in \mathbf{C}$; if $\mathbf{C}$ is order complete, that is, any bounded subset of $\mathbf{C}$ has $\vee$ and $\wedge$, then (see [6]) any regular (o)-martingale is 
(o)-convergent and

$$
\text { (o)- } \lim _{n \rightarrow+\infty} M_{n}(x)=M_{\infty}(x) \text { for } x \in \mathbf{C},
$$

where $M_{\infty}$ is the projection operator generated by the projection band $\mathbf{M}_{\infty}:=$ $\left(\bigcup_{n \geq 1} \mathbf{M}_{n}\right)^{\perp \perp}$. In addition (see also [] ) the regular (o)-martingales are the only (o)-convergent martingales.

With the above preparations, we can now formulate the following result, whose proof will be given in the last section.

Corollary 3. Under the hypothesis of Theorem 1, if $\left(\mathbf{M}_{n}\right)_{n \geq 1}$ is a filtration on $\mathbf{C}$, and $\left(x_{n}\right)_{n \geq 1}$ is an (o)-martingale with respect to $\left(\mathbf{M}_{n}\right)_{n \geq 1}$, then there exist two regular (o)-martingales $\left(y_{n}^{i}\right)_{n \geq 1}=\left(y_{n, \alpha}^{i}\right)_{n \geq 1}, \alpha \in \mathbb{R}, i=1,2$, such that

$$
x_{n}=\Phi\left(y_{n}^{1}\right)-\Phi\left(y_{n}^{2}\right) \text { for } n \geq 1,
$$

where the trading strategy operator $\Phi$ is defined in formula (1). The regular (o)martingales may be adapted to filtrations other than $\left(\mathbf{M}_{n}\right)_{n \geq 1}$.

Corollary 3 suggests an alternate definition for market completeness, using the following approximate (o)-martingale spanning, and describing the degree of density of the regular (o)-martingales within the space of all (o)-martingales, by means of the trading strategy operator $\Phi$; see formulas (1) and (3).

Definition 2. Suppose that, in a two-times economy, the space of cash flows is described by a linear lattice of functions. The subsequent market is called (o)complete iff every (o)-martingale can be expressed as the (o)-limit of regular (o)martingales, adapted to different filtrations eventually.

Remark 3. Our results show that, if the space of cash flows $\mathbf{C}$ is a countably order compete linear lattice with weak unit, then the market is (o)-complete in the sense of Definition 2. Moreover, if $\mathbf{C}$ is a $K B$-space (that is, an order complete normed linear lattice with order continuous norm, for which any norm bounded positive and order increasing sequence has $\vee$ ), such as the classical $L^{p}(\Omega, \mathcal{K}, P)$-spaces with $p \geq 1$, we proved in [7] that the regular (o)-martingales are precisely the relatively weakly compact martingales in $\mathbf{C}$ (or, equivalently, the uniformly integrable ones, by the abstract Dunford-Pettis' characterisation result). In this setting, Corollary 3 also gives a representation of (o)-martingales as strong limits of regular (o)martingales; this follows by the fact that the norm on $\mathbf{C}$ is monotone, hence the (o)-limits in formula (3) also hold in the norm sense (as well as the estimations in formula (9); see below). In particular it follows that, if $\mathbf{C}$ is a $K B$-space, then the market is (o)-complete in the sense of Definition 2, replacing the (o)-limits by norm limits.

Combining Remarks 2 and 3, we deduce that Definitions 1 and 2 for market completeness are intermediate notions between completeness in weak and norm topologies. The advantages in using these new definitions are:

a) the closed form of the admissible trading strategy (see formula (2) used in the proof of market completeness in the sense of Definition 1);

b) the probabilistic equivalence between market completeness and the approximate (o)-martingale spanning (see Definition 2 and its consequences). 


\section{ProOFS}

Proof of Theorem 1. We follow closely Freudenthal's integral representation in linear lattices (see 4], Chapter 6). First of all, as the lattice $\mathbf{C}$ is Archimedean ordered and $\lim _{\alpha \rightarrow \pm \infty} c_{\alpha}= \pm \infty$, it is easy to see that the total position $y=\left(y_{\alpha}\right)_{\alpha \in \mathbb{R}} \in \mathbf{Y}$ defined in formula (2) satisfies

$$
\vee_{\alpha} y_{\alpha}=\mathbf{1} \text { and } \wedge_{\alpha} y_{\alpha}=0 .
$$

For a partition $\left(u_{i}\right)_{i \in \mathbb{Z}}$ of $\mathbb{R}$, using (4), as $k \rightarrow+\infty$, we have

$$
\sum_{i=0}^{k}\left(y_{u_{i+1}}-y_{u_{i}}\right)=y_{u_{k+1}}-y_{0} \nearrow \mathbf{1}-y_{0} \text { and } \sum_{i=-1}^{-k}\left(y_{u_{i+1}}-y_{u_{i}}\right)=y_{0}-y_{u_{-k}} \searrow y_{0} ;
$$

summing these relations and, as $\mathbf{1}$ is a total element in $\mathbf{C}$, we deduce

$$
\sum_{i \in \mathbb{Z}}\left[y_{u_{i+1}}^{\perp \perp}(c)-y_{u_{i}}^{\perp \perp}(c)\right]=c .
$$

If $u_{i} \leq r_{i}<u_{i+1}$ are fixed sample points, straightforward algebraic computations show that we have

$$
\begin{aligned}
& \left(c_{u_{i}}-c_{r_{i}}\right)\left(y_{u_{i+1}}-y_{u_{i}}\right) \\
\leq & y_{u_{i+1}}^{\perp \perp}(c)-y_{u_{i}}^{\perp \perp}(c)-c_{r_{i}}\left(y_{u_{i+1}}-y_{u_{i}}\right) \\
\leq & \left(c_{u_{i+1}}-c_{r_{i}}\right)\left(y_{u_{i+1}}-y_{u_{i}}\right) .
\end{aligned}
$$

Now take a sequence of partitions $\Delta_{n}=\left(u_{i}^{n}\right)_{i \in \mathbb{Z}}$ of $\mathbb{R}$ and $\epsilon>0$. As $c_{\alpha}$ is continuous, there exists $\delta(\epsilon)$ such that $\left|c_{u_{i+1}^{n}}-c_{u_{i}^{n}}\right|<\left\|\Delta_{n}\right\|$ if $\left|u_{i+1}^{n}-u_{i}^{n}\right|<\delta(\epsilon)$. Choose $n_{0} \in \mathbb{N}$ such that $\max _{i \in \mathbb{Z}}\left|u_{i+1}^{n}-u_{i}^{n}\right|<\delta(\epsilon)$ for $n \geq n_{0}$ and, as $c_{\alpha}$ is increasing, we obtain $-\left\|\Delta_{n}\right\| \leq c_{u_{i}^{n}}-c_{u_{i+1}^{n}} \leq c_{u_{i}^{n}}-c_{r_{i}^{n}} \leq c_{u_{i+1}^{n}}-c_{u_{i}^{n}} \leq\left\|\Delta_{n}\right\|$. Combining with (6) gives

$$
\left|y_{u_{i+1}^{n}}^{\perp \perp}(c)-y_{u_{i}^{n}}^{\perp \perp}(c)-c_{r_{i}^{n}}\left(y_{u_{i+1}^{n}}-y_{u_{i}^{n}}\right)\right| \leq\left\|\Delta_{n}\right\|\left(y_{u_{i+1}^{n}}-y_{u_{i}^{n}}\right) .
$$

In the previous formula, sum both sides over $i \in \mathbb{Z}$ and obtain, by means of (5),

$$
\left|c-\sum_{i \in \mathbb{Z}} c_{r_{i}^{n}}\left(y_{u_{i+1}^{n}}-y_{u_{i}^{n}}\right)\right| \leq\left\|\Delta_{n}\right\| \cdot \mathbf{1} .
$$

We thus obtained that the (o)-limit in (1) makes sense for $y=\left(y_{\alpha}\right)_{\alpha \in \mathbb{R}}$ given in (2). Finally pass to (o)- $\lim _{n}$ with $\left\|\Delta_{n}\right\| \rightarrow 0$ in (7) to obtain $\Phi(y)=c$. The proof of Theorem 1 is now complete.

Proof of Corollary 3. Let us start with the following observation: if a process $\left(x_{n}\right)_{n \geq 1} \subset \mathbf{C}$ is (o)-martingale with respect to $\left(\mathbf{M}_{n}\right)_{n \geq 1}$, then it is a decreasing sequence (see also [6] for the general properties of (o)-martingales). Indeed, as $x \leq M_{n}(x)$ for $x \leq 0$, it follows that $x_{n+1} \leq M_{n}\left(x_{n+1}\right)=x_{n}$ for $n \geq 1$.

Now, let us fix an (o)-martingale $\left(x_{n}\right)_{n \geq 1}$ with respect to $\left(\mathbf{M}_{n}\right)_{n \geq 1}$, and write it as follows:

$$
x_{n}=\left(x_{n} \wedge 0\right)-\left(-\left(x_{n}\right)_{+}\right)=: x_{n}^{1}-x_{n}^{2} \text { for } n \geq 1 .
$$

Apply Theorem 1 to $\left(x_{n}^{i}\right)_{n \geq 1}$ to obtain, by formula (2), the existence of $y_{n, \alpha}^{i}:=$ $\left(c_{\alpha} \cdot \mathbf{1}-x_{n}^{i}\right)_{+}^{\perp \perp}(\mathbf{1})$, with $\lim _{\alpha \rightarrow \pm \infty} c_{\alpha}= \pm \infty$, and such that (3) holds. As $\left(x_{n}^{i}\right)_{n \geq 1}, i=$ 
1,2, are negative (o)-martingales, they are decreasing, hence for $n \geq 1$ we have $c_{\alpha} \cdot \mathbf{1}-x_{n}^{i} \leq c_{\alpha} \cdot \mathbf{1}-x_{n+1}^{i}$, that is, $\left(c_{\alpha} \cdot \mathbf{1}-x_{n}^{i}\right)_{+}^{\perp \perp} \subseteq\left(c_{\alpha} \cdot \mathbf{1}-x_{n+1}^{i}\right)_{+}^{\perp \perp}$, and

$$
\left(c_{\alpha} \cdot \mathbf{1}-x_{n}^{i}\right)_{+}^{\perp \perp}\left(\left(c_{\alpha} \cdot \mathbf{1}-x_{n+1}^{i}\right)_{+}^{\perp \perp}(\mathbf{1})\right)=\left(c_{\alpha} \cdot \mathbf{1}-x_{n}^{i}\right)_{+}^{\perp \perp}(\mathbf{1})
$$

for every $\alpha \in \mathbb{R}, i=1,2$. The latter facts show that $n \rightarrow\left(c_{\alpha} \cdot \mathbf{1}-x_{n}^{i}\right)_{+}^{\perp \perp}(\mathbf{1})$ is a regular (o)-martingale with respect to the filtration $\left(\left(c_{\alpha} \cdot \mathbf{1}-x_{n}^{i}\right)_{+}^{\perp \perp}\right)_{n \geq 1}, i=1,2$. Therefore, the proof of Corollary 3 is achieved.

\section{REFERENCES}

1. F. Delbaen and W. Schachermayer, A general version of the fundamental theorem of asset pricing, Math. Ann. 300 (1994), 463-520. MR 95m:90022

2. R.C. Green and R.A. Jarrow, Spanning and completeness in markets with contingent claims, J. Econ. Th. 41 (1987), 202-210. MR 87m:90016

3. R.A. Jarrow, X. Jin, and D.B. Madan, The second fundamental theorem of asset pricing, Math. Finance 9 (1999), 255-273.

4. W.A.J. Luxemburg and A.C. Zaanen, Riesz Spaces I, North-Holland, Amsterdam, 1971. MR 58:23483

5. D.C. Nachman, Efficient funds for meager asset spaces, J. Econ. Th. 43 (1987), 335-347. MR 88j:90029

6. G. Stoica, Order convergence and decompositions of stochastic processes, Ann. Univ. Bucharest Math. Ser. 42 (1993), 85-91. MR 95k:60111

7. G. Stoica, The structure of stochastic processes in normed vector lattices, Stud. Cerc. Mat. Rep. 46 (1994), 477-486. MR 2000b:60111

Department of Mathematics, University of New Brunswick, P.O. Box 5050, Saint John, New Brunswick, Canada E2L 4L5

E-mail address: stoica@unbsj.ca 